\title{
A Compact Variant of the QCR Method for Quadratically Constrained Quadratic 0-1 Programs
}

\author{
Laura Galli* $\quad$ Adam N. Letchford ${ }^{\dagger}$ \\ To appear in Optimization Letters
}

\begin{abstract}
Quadratic Convex Reformulation (QCR) is a technique that was originally proposed for quadratic $0-1$ programs, and then extended to various other problems. It is used to convert non-convex instances into convex ones, in such a way that the bound obtained by solving the continuous relaxation of the reformulated instance is as strong as possible.

In this paper, we focus on the case of quadratically constrained quadratic 0-1 programs. The variant of QCR previously proposed for this case involves the addition of a quadratic number of auxiliary continuous variables. We show that, in fact, at most one additional variable is needed. Some computational results are also presented.
\end{abstract}

Keywords: Combinatorial optimization, Semidefinite programming, Quadratically constrained quadratic programming

\section{Introduction}

It has been known for some time that semidefinite programming (SDP) can be used to derive strong convex relaxations of various hard quadratic optimisation problems. This includes, for example, quadratic zero-one programming (0-1 QP) $[12,16]$, non-convex quadratically constrained quadratic programming (QCQP) $[10,17,20]$ and 0-1 QCQP $[8,14,18]$.

In a recent paper, Billionnet et al. [6] proposed to use SDP to reformulate 0-1 QP instances, rather than merely relax them. Their method, called Quadratic Convex Reformulation (QCR), has two effects. First, it converts non-convex instances into convex ones. Second, when applied to instances that are already convex, it improves the bound obtained by solving the continuous relaxation of the instance. Once QCR has been applied, the

\footnotetext{
*Dipartimento di Informatica, Università di Pisa, Largo B. Pontecorvo 3, 56127 Pisa, Italy. E-mail: Laura.Galli@di.unipi.it

${ }^{\dagger}$ Corresponding author. Department of Management Science, Lancaster University, Lancaster LA1 4YX, United Kingdom. E-mail: A.N.Letchford@lancaster.ac.uk
} 
reformulated instance can be fed into any software package capable of solving convex 0-1 QP instances.

Recently, Billionnet et al. $[4,5]$ have extended QCR to mixed-integer quadratic programming (MIQP) and mixed-integer quadratically constrained quadratic programming (MIQCQP). The purpose of the present paper is to present a much simpler variant of QCR for the case of quadratically constrained quadratic 0-1 programming (0-1 QCQP).

The structure of the paper is as follows. In Section 2, we review the relevant literature. In Section 3, we present our extension of QCR. In Section 4 , we present some computational results and some concluding remarks.

\section{Literature Review}

We now review the relevant literature. We cover SDP and Lagrangian relaxations for non-convex QCQP in Subsection 2.1, their application to 0-1 QP in Subsection 2.2, and the QCR method in Subsection 2.3.

\subsection{Relaxations of non-convex QCQP}

A general instance of QCQP can be written in the following form:

$$
\begin{array}{cc}
\text { inf } & x^{T} Q^{0} x+c^{0} \cdot x \\
\text { s.t. } & x^{T} Q^{j} x+c^{j} \cdot x \leq h_{j} \quad(j=1, \ldots, m) \\
& x \in \mathbb{R}^{n},
\end{array}
$$

where the $Q^{j}$ are symmetric matrices of order $n$, the $c^{j}$ are $n$-vectors and the $h_{j}$ are scalars. (We write 'inf' rather than 'min' because it is possible that the infimum is not attainable.)

Now suppose that at least one of the $Q^{j}$ is not positive semidefinite (psd), so that the problem is not convex. We can derive a SDP relaxation as follows $[10,17,20]$. We define the $n \times n$ matrix $X=x x^{T}$, along with the augmented matrix

$$
Y=\left(\begin{array}{l}
1 \\
x
\end{array}\right)\left(\begin{array}{l}
1 \\
x
\end{array}\right)^{T}=\left(\begin{array}{cc}
1 & x^{T} \\
x & X
\end{array}\right)
$$

Note that $Y$ is symmetric and psd. The following SDP is therefore a relaxation of non-convex QCQP:

$$
\begin{array}{cc}
\text { inf } & Q^{0} \bullet X+c^{0} \cdot x \\
\text { s.t. } & Q^{j} \bullet X+c^{j} \cdot x \leq h_{j} \quad(j=1, \ldots, m) \\
& Y \succeq 0 .
\end{array}
$$

Here, $Q^{j} \bullet X$ denotes $\sum_{i=1}^{n} \sum_{k=1}^{n} Q_{i k}^{j} X_{i k}$, and $Y \succeq 0$ means that $Y$ is symmetric and psd. 
There is a connection between SDP and Lagrangian relaxations of nonconvex QCQP $[9,10,14,16]$. Suppose we relax the constraints (1) in Lagrangian fashion, using a vector $\lambda \in \mathbb{R}_{+}^{m}$ of Lagrangian multipliers. The Lagrangian is:

$$
f(x, \lambda)=x^{T}\left(Q^{0}+\sum_{j=1}^{m} \lambda_{j} Q^{j}\right) x+\left(c^{0}+\sum_{j=1}^{m} \lambda_{j} c^{j}\right) \cdot x-\sum_{j=1}^{m} \lambda_{j} h_{j} .
$$

The relaxed problem is:

$$
\inf \left\{f(x, \lambda): x \in \mathbb{R}^{n}\right\},
$$

which is an unconstrained quadratic minimisation problem. The Lagrangian dual is:

$$
\sup _{\lambda \in \mathbb{R}_{+}^{m}} \inf _{x \in \mathbb{R}^{n}} f(x, \lambda) .
$$

As explained in $[10,14,16]$, if the supremum is attainable by some multiplier vector $\lambda^{*}$, then $\lambda^{*}$ must be an optimal dual solution to the SDP. If in addition strong duality holds for the SDP, then the semidefinite and Lagrangian bounds will be equal. An analogous result holds when quadratic equations, rather than inequalities, are present.

\subsection{Relaxations of 0-1 QP}

An instance of 0-1 QP can be written in the following form:

$$
\begin{array}{cc}
\min & x^{T} Q x+c^{T} x \\
\text { s.t. } & A x=b \\
& D x \leq f \\
& x \in\{0,1\}^{n},
\end{array}
$$

where $Q$ is again a symmetric square matrix and $A, D, c, b$ and $f$ are matrices and vectors of appropriate dimension.

As observed by many authors (e.g., [11, 13, 14, 15, 16]), the condition that $x$ be binary is equivalent to the non-convex quadratic constraints

$$
x_{i}^{2}-x_{i}=0 \quad(i=1, \ldots, n) .
$$

That is, 0-1 QP can be regarded as a special case of non-convex QCQP. This observation suggests immediately the following SDP relaxation:

$$
\inf \left\{Q \bullet X+c^{T} x:(2),(3), x=\operatorname{diag}(X), Y \succeq 0\right\} .
$$

The SDP can be strengthened using some ideas presented in $[15,19]$. Given a linear equation in the system $(2)$, say $a^{j} \cdot x=b_{j}$, and any variable, 
say $x_{k}$, the quadratic equation $\left(a^{j} \cdot x\right) x_{k}=b_{j} x_{k}$ is satisfied by all feasible solutions. This implies that the equation

$$
\sum_{i=1}^{n} a_{i}^{j} X_{i k}-b_{j} x_{k}=0
$$

can be added to the SDP. In a similar way, any linear inequality in the system (3) can be multiplied by either $x_{k}$ or $1-x_{k}$ to yield valid quadratic inequalities, which can also be converted into valid inequalities for the SDP. For further ways of strengthening the SDP, see, e.g., [9, 12, 14, 16, 18].

It follows from the result mentioned in Subsection 2.1 that, for any SDP relaxation of 0-1 QP, there is a corresponding Lagrangian relaxation. Examples of such relaxations appear, for example, in $[10,13,14,16]$.

\subsection{The QCR method}

The QCR method has its roots in an early paper by Hammer \& Rubin [11]. They proposed to convert non-convex 0-1 QP instances into convex ones, simply by subtracting $\sum_{1 \leq i \leq n} \alpha\left(x_{i}^{2}-x_{i}\right)$ from the objective, where $\alpha$ is the minimum eigenvalue of $Q$. Note that this reformulation leaves the cost of every feasible solution unchanged.

Billionnet \& Elloumi [3] applied a similar idea to unconstrained 0-1 QP. They perturbed the objective by adding terms of the form $\lambda_{i}\left(x_{i}^{2}-x_{i}\right)$, where $\lambda \in \mathbb{R}^{n}$, in such a way that (i) the resulting objective function is convex, and (ii) the lower bound obtained by solving the continuous relaxation of the instance is maximised.

Billionnet et al. [6] extended this approach to the case of equalityconstrained 0-1 QP. They perturbed the objective not only by adding terms of the form $\lambda_{i}\left(x_{i}^{2}-x_{i}\right)$, but also by adding terms of the form $M_{j k}\left(a^{j} \cdot x-b_{j}\right) x_{k}$, for some constraint index $j$ and variable index $k$. To do this, they proposed to solve the SDP mentioned in the previous subsection, strengthened with the constraints of the form (4), and then set $\lambda$ and $M$ to the optimal dual values for the constraints $\operatorname{diag}(X)=x$ and (4), respectively. This is the original version of QCR.

As mentioned in the introduction, Billionnet et al. [4, 5] extended QCR to instances of MIQP and MIQCQP satisfying certain technical conditions. For the sake of brevity, we do not go into further details. We remark however that their approaches involve the addition of $\mathcal{O}\left(n^{2}\right)$ variables. Our approach to $0-1$ QCQP works with the original $n$ variables only.

We close this section with three remarks. First, some alternative heuristics for selecting the multipliers $\lambda_{i}$ appeared in [1]. They involve either minimising the trace of the perturbed matrix, or minimising the maximum eigenvalue. Second, some alternative approaches to MIQCQP were surveyed in [8]. Finally, QCR was very recently extended to QP with linear complementarity constraints in [2]. 


\section{Theoretical Results}

In this section, we show how to extend QCR to general a 0-1 QCQP instance of the form:

$$
\begin{array}{ccl}
\min & x^{T} Q^{0} x+c^{0} \cdot x & \\
\text { s.t. } & x_{i}^{2}-x_{i}=0 & (i=1, \ldots, n) \\
& x^{T} Q^{j} x+c^{j} \cdot x=h_{j} & (j=1, \ldots, m) . \\
& x^{T} Q^{j} x+c^{j} \cdot x \leq h_{j} & (j=m+1, \ldots, m+r) .
\end{array}
$$

For simplicity of notation, we assume that any linear constraints, or any additional valid quadratic constraints generated from them (as in Subsection 2.2 ), are already included in the systems (7) and (8).

It turns out that quadratic inequalities are harder to handle than quadratic equations. We therefore deal with equations only in Subsection 3.1, before moving on to the general case in Subsection 3.2.

\subsection{The equality-constrained case}

Consider an equality-constrained 0-1 QCQP instance of the form (5)-(7). The continuous relaxation of this instance is obtained by replacing the binary conditions (6) with the weaker conditions $0 \leq x_{i} \leq 1$ for all $i$.

We wish to 'convexify' the instance, which in this context means transforming the instance into an equivalent 0-1 QCQP instance (which will turn out to be inequality-constrained) that has the following four properties:

- The objective function is convex.

- The constraint functions are all convex.

- The set of feasible solutions is unchanged.

- The cost of each feasible solution is unchanged.

Ideally, we would like the lower-bound from the continuous relaxation of the transformed instance to equal the lower bound from the following SDP relaxation:

$$
\begin{array}{cc}
\text { inf } & Q^{0} \bullet X+c^{0} \cdot x \\
\text { s.t. } & \operatorname{diag}(X)=x \\
& Q^{j} \bullet X+c^{j} \cdot x=h_{j} \quad(j=1, \ldots, m) \\
& Y \succeq 0 .
\end{array}
$$

Moreover, in contrast to Billionnet et al. [5], we would like to do this without using additional variables.

Observe that, when perturbing the objective function, we can add terms of the following two forms: 
1. $x^{T} \operatorname{Diag}(\lambda) x-\lambda^{T} x$ for some $\lambda \in \mathbb{R}^{n}$

2. $\mu_{j}\left(x^{T} Q^{j} x+c^{j} \cdot x-h_{j}\right)$ for some $\mu \in \mathbb{R}^{m}$.

The following theorem shows that, using these two sources of perturbation, the desired reformulation can be obtained:

Theorem 1 Let a 0-1 QCQP instance of the form (5)-(7) be given. Suppose that strong duality holds for the SDP (9)-(12), and that a dual optimal solution exists. Let $\left(\lambda^{*}, \mu^{*}\right)$ be such a solution, where $\lambda$ and $\mu$ are the dual variables for the constraints (10)-(11). Then, suppose we perform the following three operations:

- perturb the objective function of the 0-1 QCQP instance by adding terms of the form $x^{T} \operatorname{Diag}\left(\lambda^{*}\right) x-\left(\lambda^{*}\right)^{T} x$ and $\mu_{j}^{*}\left(x^{T} Q^{j} x+c^{j} \cdot x-h_{j}\right)$ for $j=1, \ldots, m$;

- replace each of the quadratic equations (7) with two quadratic inequalities of opposite sign;

- convexify the resulting quadratic inequalities using any desired method (such as the minimum eigenvalue method).

Then the reformulated 0-1 QCQP instance will be convex, and the lower bound from its continuous relaxation will equal the SDP lower bound.

Proof. From the equivalence of semidefinite and Lagrangian relaxations described in Subsection 2.1, the lower bound from the SDP will be identical to the lower bound from the Lagrangian dual under the stated conditions. Moreover, the optimal Lagrangian multipliers for the constraints (6)-(7) will be equal to $\lambda^{*}$ and $\mu^{*}$, respectively. So, the lower bound from the Lagrangian dual will be equal to:

$$
\min \left\{x^{T} \bar{Q} x+\bar{c} \cdot x+\bar{h}: x \in \mathbb{R}^{n}\right\},
$$

where

$$
\begin{aligned}
\bar{Q} & =Q^{0}+\operatorname{Diag}\left(\lambda^{*}\right)+\sum_{j=1}^{m} \mu_{j}^{*} Q^{j} \\
\bar{c} & =c^{0}-\lambda^{*}+\sum_{j=1}^{m} \mu_{j}^{*} c^{j} \\
\bar{h} & =-\sum_{j=1}^{m} \mu_{j}^{*} h_{j} .
\end{aligned}
$$

Now observe that the objective function of (13) is identical to the objective function of the reformulated instance. Therefore, the lower bound from the 
continuous relaxation is at least as large as (13). The continuous relaxation has some constraints that are not present in (13); namely, the convexified quadratic inequalities and the constraint $x \in[0,1]^{n}$. But these constraints cannot improve the bound, since they are convex and are implied by constraints that have already been incorporated into the objective function of (13) with optimal multipliers.

It remains to be shown that the reformulated instance is convex. Since $\lambda^{*}$ and $\mu^{*}$ belong to a feasible dual solution, we have $\bar{Q} \succeq 0$, and therefore the objective function is convex. Moreover, the constraints are convex by construction.

Notice that, for Theorem 1 to be applicable, strong duality must hold and a dual optimal solution must exist. The following proposition implies that strong duality does indeed hold:

Proposition 1 The dual of the SDP (9)-(12) satisfies the Slater condition (i.e., is strictly feasible).

Proof. The dual can be written in the following form (see, e.g., $[6,14])$ :

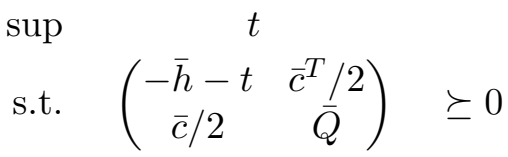

$$
\begin{aligned}
& (\lambda, \mu, t) \in \mathbb{R}^{n+m+1},
\end{aligned}
$$

where $\bar{Q}, \bar{c}$ and $\bar{h}$ are defined as above. (Here, $t$ is the dual variable for the constraint $Y_{00}=1$, which is implicit in the definition of $Y$.) Given any $\mu$, we can ensure that the matrix

$$
\left(\begin{array}{cc}
-\bar{h}-t & \bar{c}^{T} / 2 \\
\bar{c} / 2 & \bar{Q}
\end{array}\right)
$$

is positive definite by decreasing $t$ and increasing the components of $\lambda$ by large enough amounts.

Unfortunately, due to the unboundedness of the dual SDP, a dual optimal solution is not guaranteed to exist (see also [9]). Fortunately, we have found that such a solution can almost always be found in practice.

\subsection{The inequality-constrained case}

We now move on to the general case, in which inequalities may be present. The SDP for this case is obtained simply by adding the following constraints to $(9)-(12)$ :

$$
Q^{j} \bullet X+c^{j} \cdot x \leq h_{j} \quad(j=m+1, \ldots, m+r) .
$$


It turns out, however, that we cannot always reformulate an inequalityconstrained 0-1 QCQP instance in such a way that the lower bound from the continuous relaxation is equal to the SDP bound. In fact, the best possible lower bound can be much worse than the SDP bound. This is illustrated by the following example:

Example: Consider the following 0-1 QCQP instance:

$$
\begin{array}{ccl}
\min & -\sum_{i=1}^{n} x_{i} & \\
\mathrm{s.t.} & x_{i}^{2}-x_{i}=0 & (i=1, \ldots, n) \\
& x_{i} x_{j} \leq 0 & (1 \leq i<j \leq n) .
\end{array}
$$

The optimal profit is -1 , and the lower bounds from the primal and dual SDPs are easily shown to be -1 as well. As for QCR, there is no point perturbing the objective function using the equations (15), since negative values for the multipliers $\lambda_{i}$ would destroy convexity and positive values would weaken the lower bound. On the other hand, the quadratic inequalities (16) are non-convex, and therefore must be convexified using the equations (15). By symmetry, there exists an optimal reformulation in which the inequalities (16) are replaced by inequalities of the form:

$$
\alpha\left(x_{i}^{2}-x_{i}\right)+\alpha\left(x_{j}^{2}-x_{j}\right)+x_{i} x_{j} \leq 0 \quad(1 \leq i<j \leq n),
$$

for some real $\alpha$. For convexity, we require $\alpha \geq 1 / 2$. The best lower bound is obtained when $\alpha=1 / 2$. Then, the optimal solution $x^{*}$ to the continuous relaxation is $(1 / 2, \ldots, 1 / 2)^{T}$, yielding a lower bound of $-n / 2$.

This example also illustrates the fact that, to find an optimal reformulation, one needs to find an optimal perturbation of each quadratic inequality, and the objective function, simultaneously. It can be shown that is possible to find these perturbations by solving a large SDP, involving a matrix variable of order $(n+1) r$. We do not go into details, however, since we do not recommend such an approach.

A natural alternative way to handle quadratic inequalities is to convert them into equations, by adding (continuous and non-negative) slack variables. One can then apply the reformulation scheme presented in the previous subsection. It can be shown that, if this is done, the lower bound for the resulting mixed 0-1 QCQP instance is equal to the SDP bound for the original 0-1 QCQP instance. This does however mean that $r$ new variables have to be added.

In fact, it is possible to use just one slack variable, as shown in the following proposition:

Proposition 2 Let a 0-1 QCQP instance of the form (5)-(8) be given, and suppose as before that a dual optimal SDP solution $\left(\lambda^{*}, \mu^{*}, \nu^{*}\right)$ exists. Then, suppose we perform the following four operations: 
- add the following constraint to the 0-1 QCQP instance:

$$
\sum_{j=m+1}^{m+r} \nu_{j}^{*}\left(x^{T} Q^{j} x+c^{j} \cdot x\right)+s=\sum_{j=m+1}^{m+r} \nu_{j}^{*} h_{j},
$$

where $s$ is a new continuous and non-negative slack variable;

- perturb the objective function of the resulting mixed 0-1 QCQP instance by adding the variable $s$, along with terms of the form $x^{T} \operatorname{Diag}\left(\lambda^{*}\right) x-$ $\left(\lambda^{*}\right)^{T} x, \mu_{j}^{*}\left(x^{T} Q^{j} x+c^{j} \cdot x-h_{j}\right)$ for $j=1, \ldots, m$, and $\nu_{j}^{*}\left(x^{T} Q^{j} x+c^{j}\right.$. $\left.x-h_{j}\right)$ for $j=m+1, \ldots, m+r$;

- replace each quadratic equation with two quadratic inequalities;

- convexify all quadratic inequalities using any desired method.

Then the reformulated mixed 0-1 QCQP instance will be convex, and the lower bound from its continuous relaxation will be equal to the SDP bound for the original 0-1 QCQP instance.

Proof. Observe that the equation (17) is obtained by multiplying each of the $r$ quadratic inequalities (8) by its corresponding $\nu^{*}$ value, summing the resulting inequalities together, and adding a slack variable. Since $\nu^{*}$ is by assumption part of a dual optimal solution, the SDP bound for the original instance would remain unchanged if we replaced the $r$ inequalities (8) with the single equation (17). The rest of the proof is similar to that of Theorem 1.

\section{Computational Results}

We now present the results of some computational experiments. We created six sets of random 0-1 QCQP instances of the following form:

$$
\max \left\{c^{0} \cdot x+x^{T} Q^{0} x: c^{j} \cdot x+x^{T} Q^{j} x \leq b^{j}, x \in\{0,1\}^{n} j=1 \ldots m\right\},
$$

where $c^{0}, c^{j}, Q^{0}$ and $Q^{j}$ all have positive integer components, and $b^{j}$ is set to:

$$
\left\lceil\frac{1}{2} \sum_{i=1}^{n} c_{i}^{j}+\frac{1}{4} \sum_{i=1}^{n} \sum_{j=1}^{n} Q_{i j}^{j}\right\rceil .
$$

Note that the optimal profit is guaranteed to be positive for such instances.

Each set of instances has $n \in\{5,10, \ldots, 30\}$, and is created by varying three parameters: $m$, the number of constraints; $\Delta$, the density of the constraint matrix (i.e., the percentage of non-zero entries), and $r$, the range of the non-zero coefficients. See the first four columns of Table 1 for details.

For the instances with $m=1$, we applied five reformulation schemes: 
Table 1: Gaps and computing times (in seconds) for five reformulation schemes

\begin{tabular}{|c|c|c|c|c|c|c|c|c|c|c|c|c|c|}
\hline \multirow[b]{2}{*}{$n$} & \multirow[b]{2}{*}{$m$} & \multirow[b]{2}{*}{$\Delta$} & \multirow[b]{2}{*}{$r$} & \multicolumn{2}{|c|}{ Scheme1 } & \multicolumn{2}{|c|}{ Scheme2 } & \multicolumn{2}{|c|}{ Scheme3 } & \multicolumn{2}{|c|}{ Scheme4 } & \multicolumn{2}{|c|}{ Scheme5 } \\
\hline & & & & $\%$ gap & time & $\%$ gap & time & $\%$ gap & time & $\%$ gap & time & $\%$ gap & time \\
\hline 5 & 1 & 100 & {$[1,100]$} & 133.96 & 0.00 & 116.98 & 0.01 & 119.36 & 0.00 & 116.98 & 0.00 & 44.37 & 0.00 \\
\hline 10 & 1 & 100 & {$[1,100]$} & 82.43 & 0.00 & 74.90 & 0.01 & 76.13 & 0.00 & 74.90 & 0.01 & 18.34 & 0.00 \\
\hline 15 & 1 & 100 & {$[1,100]$} & 59.20 & 0.01 & 52.99 & 0.01 & 54.59 & 0.01 & 52.99 & 0.01 & 5.53 & 0.01 \\
\hline 20 & 1 & 100 & {$[1,100]$} & 69.29 & 0.02 & 64.65 & 0.03 & 66.19 & 0.02 & 64.65 & 0.02 & 5.03 & 0.02 \\
\hline 25 & 1 & 100 & {$[1,100]$} & 64.65 & 0.02 & 62.34 & 0.03 & 63.53 & 0.02 & 62.34 & 0.02 & 6.77 & 0.02 \\
\hline 30 & 1 & 100 & {$[1,100]$} & 66.03 & 0.03 & 63.02 & 0.03 & 63.65 & 0.03 & 63.01 & 0.03 & 3.57 & 0.03 \\
\hline 5 & 1 & 100 & {$[1,500]$} & 136.60 & 0.00 & 129.54 & 0.01 & 130.86 & 0.01 & 129.54 & 0.01 & 47.73 & 0.01 \\
\hline 10 & 1 & 100 & {$[1,500]$} & 62.98 & 0.01 & 56.96 & 0.01 & 59.03 & 0.01 & 56.88 & 0.01 & 15.03 & 0.01 \\
\hline 15 & 1 & 100 & {$[1,500]$} & 49.91 & 0.03 & 45.62 & 0.03 & 46.77 & 0.01 & 45.62 & 0.02 & 9.38 & 0.01 \\
\hline 20 & 1 & 100 & {$[1,500]$} & 55.47 & 0.03 & 52.94 & 0.03 & 54.08 & 0.02 & 52.93 & 0.03 & 3.04 & 0.01 \\
\hline 25 & 1 & 100 & {$[1,500]$} & 68.82 & 0.03 & 65.57 & 0.03 & 66.77 & 0.02 & 65.57 & 0.03 & 4.95 & 0.02 \\
\hline 30 & 1 & 100 & {$[1,500]$} & 65.77 & 0.04 & 64.15 & 0.04 & 64.64 & 0.03 & 64.15 & 0.05 & 4.09 & 0.02 \\
\hline 5 & 1 & 50 & {$[1,100]$} & 106.52 & 0.00 & 81.65 & 0.01 & 92.25 & 0.01 & 81.59 & 0.01 & 63.04 & 0.01 \\
\hline 10 & 1 & 50 & {$[1,100]$} & 43.60 & 0.01 & 28.87 & 0.01 & 36.12 & 0.01 & 28.78 & 0.01 & 8.35 & 0.01 \\
\hline 15 & 1 & 50 & {$[1,100]$} & 38.09 & 0.03 & 33.39 & 0.01 & 36.02 & 0.01 & 32.69 & 0.02 & 7.92 & 0.01 \\
\hline 20 & 1 & 50 & {$[1,100]$} & 32.59 & 0.03 & 25.96 & 0.03 & 31.58 & 0.02 & 25.62 & 0.02 & 4.96 & 0.02 \\
\hline 25 & 1 & 50 & {$[1,100]$} & 46.07 & 0.03 & 39.62 & 0.03 & 43.55 & 0.02 & 39.50 & 0.04 & 6.09 & 0.02 \\
\hline 30 & 1 & 50 & {$[1,100]$} & 55.87 & 0.05 & 48.99 & 0.03 & 54.67 & 0.03 & 48.98 & 0.05 & 6.41 & 0.03 \\
\hline 5 & 5 & 100 & {$[1,100]$} & 97.01 & 0.01 & 86.06 & 0.01 & 95.87 & 0.01 & - & - & 35.07 & 0.00 \\
\hline 10 & 5 & 100 & {$[1,100]$} & 82.75 & 0.02 & 77.09 & 0.02 & 80.06 & 0.02 & - & - & 11.94 & 0.01 \\
\hline 15 & 5 & 100 & {$[1,100]$} & 87.86 & 0.02 & 83.00 & 0.02 & 84.94 & 0.03 & - & - & 18.09 & 0.02 \\
\hline 20 & 5 & 100 & {$[1,100]$} & 74.11 & 0.03 & 70.40 & 0.03 & 72.07 & 0.03 & - & - & 6.01 & 0.04 \\
\hline 25 & 5 & 100 & {$[1,100]$} & 79.09 & 0.06 & 75.80 & 0.05 & 77.22 & 0.04 & - & - & 9.32 & 0.06 \\
\hline 30 & 5 & 100 & {$[1,100]$} & 74.32 & 0.09 & 71.61 & 0.07 & 73.08 & 0.06 & - & - & 3.19 & 0.06 \\
\hline 5 & 5 & 50 & {$[1,100]$} & 217.57 & 0.00 & 182.08 & 0.00 & 196.57 & 0.00 & - & - & 90.66 & 0.00 \\
\hline 10 & 5 & 50 & {$[1,100]$} & 114.57 & 0.01 & 101.84 & 0.01 & 107.36 & 0.01 & - & - & 33.49 & 0.01 \\
\hline 15 & 5 & 50 & {$[1,100]$} & 99.86 & 0.01 & 94.75 & 0.01 & 96.94 & 0.01 & - & - & 17.24 & 0.02 \\
\hline 20 & 5 & 50 & {$[1,100]$} & 68.47 & 0.03 & 59.51 & 0.04 & 65.64 & 0.04 & - & - & 12.30 & 0.04 \\
\hline 25 & 5 & 50 & {$[1,100]$} & 71.06 & 0.06 & 63.56 & 0.05 & 68.96 & 0.08 & - & - & 12.83 & 0.06 \\
\hline 30 & 5 & 50 & {$[1,100]$} & 65.04 & 0.08 & 56.92 & 0.07 & 63.39 & 0.08 & - & - & 7.11 & 0.08 \\
\hline 5 & 10 & 50 & {$[1,100]$} & 460.40 & 0.01 & 325.88 & 0.00 & 363.92 & 0.02 & - & - & 229.79 & 0.01 \\
\hline 10 & 10 & 50 & {$[1,100]$} & 72.39 & 0.02 & 57.23 & 0.03 & 72.09 & 0.03 & - & - & 21.16 & 0.01 \\
\hline 15 & 10 & 50 & {$[1,100]$} & 81.53 & 0.04 & 73.31 & 0.06 & 79.00 & 0.06 & - & - & 19.06 & 0.03 \\
\hline 20 & 10 & 50 & {$[1,100]$} & 85.84 & 0.07 & 76.29 & 0.10 & 83.33 & 0.07 & - & - & 21.39 & 0.08 \\
\hline 25 & 10 & 50 & {$[1,100]$} & 72.33 & 0.20 & 64.29 & 0.19 & 69.60 & 0.20 & - & - & 11.37 & 0.12 \\
\hline 30 & 10 & 50 & {$[1,100]$} & 67.80 & 0.27 & 59.54 & 0.31 & 66.31 & 0.29 & - & - & 7.22 & 0.23 \\
\hline
\end{tabular}


1. Convexify the objective and constraint independently, using the minimum eigenvalue method in [11].

2. As above, but using the minimum trace method in [1].

3. As above, but using the min-max eigenvalue method in [1].

4. Best reformulation without the slack variable (see Subsection 3.2).

5. Best reformulation with slack variable (see Subsection 3.2).

For the instances with $m \in\{5,10\}$, we applied all reformulations except the fourth, which turned out to be problematic due to the huge size of the resulting SDP. The eigenvalues were computed using the $\mathrm{C}$ function Jacobi_Cyclic_Method, which is available in the Mathematics Source Library at:

http://www . mymathlib.com/matrices/eigen/symmetric.html

The SDPs were solved using the routine CSDP, due to Borchers [7], which is available as part of COIN-OR at:

$$
\text { http://projects.coin-or.org/Csdp }
$$

We also solved the instances to proven optimality using IBM CPLEX v. 12.5. (On our machine, we were unable to solve larger instances to optimality.)

Table 1 shows, for each instance and each scheme, the gap between the upper bound and the optimum, expressed as a percentage of the optimum, and the corresponding time in seconds.

It is apparent that the 'minimum trace' bound is slightly better than the 'min-max-eigenvalue' bound, which in turn is slightly better than the 'minimum eigenvalue' bound. Also, the best bound obtainable without a slack variable is only very slightly better than the 'minimum trace' bound. The bound obtained with the slack variable, on the other hand, is much stronger. This is in accordance with the results in Subsection 3.2.

One also sees that the time taken to perform the reformulation was negligible in all cases. We wanted also to record the time taken actually to solve the reformulated instances via branch-and-bound. Unfortunately, CPLEX seems to perform an internal reformulation scheme of its own prior to applying branch-and-bound, which we were unable to deactivate. We are sure, however, that the branch-and-bound times would be considerably larger than the reformulation times (minutes or hours rather than fractions of a second).

We leave to future research a full comparison between our scheme and that of $[5]$.

Acknowledgement: The second author was partially supported by the Engineering and Physical Sciences Research Council under grant EP/D072662/1. 


\section{References}

[1] K.M. Anstreicher, M. Fampa, J. Lee \& J. Williams. Using continuous nonlinear relaxations to solve constrained maximum-entropy sampling problems. Math. Program., 85, 221-240, 1999.

[2] L. Bai, J.E. Mitchell \& J.-S. Pang (2013) Using quadratic convex reformulation to tighten the convex relaxation of a quadratic program with complementarity constraints. Published online in Optim. Lett., May 2013 (doi:10.1007/s11590-013-0647-0).

[3] A. Billionnet \& S. Elloumi. Using a mixed-integer quadratic programming solver for the unconstrained quadratic 0-1 Problem. Math. Program., 109, 55-68, 2007.

[4] A. Billionnet, S. Elloumi \& A. Lambert. Extending the QCR method to general mixed-integer programs. Math. Program., 131, 381-401, 2012.

[5] A. Billionnet, S. Elloumi \& A. Lambert. Convex reformulations of integer quadratically constrained problems. Talk presented at the International Symposium on Mathematical Programming (ISMP), August 2012.

[6] A. Billionnet, S. Elloumi \& M.-C. Plateau. Improving the performance of standard solvers for quadratic 0-1 programs by a tight convex reformulation: the QCR method. Discr. Appl. Math., 157, 1185-1197, 2009 .

[7] B. Borchers. CSDP, a C Library for Semidefinite Programming. Optim. Meth. Soft., 11, 613-623, 1999.

[8] S. Burer \& A. Saxena. The MILP road to MIQCP. In: J. Lee \& S. Leyffer (eds.) Mixed-Integer Nonlinear Programming, pp. 373-406. IMA Volumes in Mathematics and its Applications, vol. 154. Berlin: Springer, 2011.

[9] A. Faye \& F. Roupin. Partial Lagrangian relaxation for general quadratic programming. 4OR, 5, 75-88, 2007.

[10] T. Fujie \& M. Kojima. Semidefinite programming relaxation for nonconvex quadratic programs. J. Glob. Opt., 10, 367-380, 1997.

[11] P.L. Hammer \& A.A. Rubin Some remarks on quadratic programming with 01 variables. RAIRO, 3, 67-79, 1970.

[12] C. Helmberg \& F. Rendl. Solving quadratic $(0,1)$-problems by semidefinite programs and cutting planes. Math. Program., 82, 291-315, 1998. 
[13] F. Körner. On duality for Boolean programming. Eur. J. Oper. Res., 46, 399-403, 1990.

[14] C. Lemaréchal \& F. Oustry. SDP relaxations in combinatorial optimization from a Lagrangian viewpoint. In N. Hadjisawas \& P.M. Pardalos (eds.), Advances in Convex Analysis and Global Optimization. Dortrecht: Kluwer, 2001.

[15] L. Lovász \& A.J. Schrijver. Cones of matrices and set-functions and 0-1 optimization. SIAM J. Optimization, 1, 166-190.

[16] S. Poljak, F. Rendl \& H. Wolkowicz (1995) A recipe for semidefinite relaxation for (0,1)-quadratic programming. J. Global Opt., 7, 51-73, 1991.

[17] M. Ramana. An Algorithmic Analysis of Multiquadratic and Semidefinite Programming Problems. PhD thesis, Johns Hopkins University, Baltimore, MD, 1993.

[18] F. Roupin. From linear to semidefinite programming: an algorithm to obtain semidefinite relaxations for bivalent quadratic problems. $J$. Comb. Opt., 8, 469-493, 2004.

[19] H.D. Sherali \& W.P. Adams. A Reformulation-Linearization Technique for Solving Discrete and Continuous Nonconvex Problems. Dordrecht: Kluwer, 1998.

[20] N. Shor. Quadratic optimization problems. Sov. J. Comput. Syst. Sci., 25, 111, 1987. 